\title{
Zum Wassertransport in den Alpen während der frühen Neuzeit
}

\begin{abstract}
During the early modern period, the transport of goods and passengers on ships and rafts was widespread not only in the lowlands, but also in the Alps. The waterway played an important role for transporting heavy loads. It was commonly used for regional trade and could also be part of the transalpine trafic. However, the ability of rivers to carry ships or rafts was not a "natural" one but was human-generated. In a changing economical, technical and political environment, this ability was a matter of constant reproduction. This is especially the case in the Alps, where every waterway had an upper limit.
\end{abstract}

\section{Résumé \\ Pendant la période moderne, le transport des marchandises et passagers par bateaux et radeaux était répandu non seule- ment dans le plays plat, mais aussi dans les Alpes. La voie d'eau y jouait un rôle important au transport des biens pon- déreuses. II servait au commerce régional et quelque part aussi au trafic transalpin. La navigabilité ou flottabilité des rivières n'était pas un avantage naturel, mais fait par l'hom- me lui-même. Quand le contexte économique, technique et politique se changeait constamment, la question de main- tenir la navigabilité ou flottabilité se posait toujours de nou- veau. Dans les Alpes, les limites de toutes les voies d'eau, soient les limites économiques de les maintenir, devenaient particulièrement visible.}

\section{Einleitung}

Die Alpen als historischer Verkehrsraum sind in der Geschichtswissenschaft wie auch in der Geographie seit langer Zeit ein Thema (z. B. BERGIER 1974/75). Im Vordergrund stehen in der Geographie die Routen und Wege, in der Geschichte oft die politischen, rechtlichen und wirtschaftlichen Aspekte, vor allem des Transitverkehrs. In den meisten Arbeiten erscheint der Wasserweg nur am Rande. Offenbar wird nicht erwartet, daß er in den Alpen mehr als nur eine marginale Rolle im Verkehrswesen früherer Jahrhunderte gespielt haben könnte. Gleichzeitig gilt aber als selbstverständlich, daß der Wasserverkehr auf den Transitrouten über die Alpenrandseen in der Lage war, fast den gesamten Güter- und Personenverkehr abzuwickeln.

Der vorliegende Artikel beruht auf einer umfangreichen Untersuchung der Schiffahrt und Flößerei (d. h. Fahrt mit meist beladenen, aus zusammengebundenen Baumstämmen bestehenden Fahrzeugen) in den Alpen während der frühen Neuzeit (BRÖNNIMANN 1997). Hier wird diese Inventarsierung von Hinweisen aus der Literatur kurz zusammengefaßt und dann eine These zum Fragenkomplex anhand eines Beispiels näher erläutert.

\section{Ein Inventar}

Das Inventar der schiff- und flößbaren Gewässer in den Alpen zeigt, daß Schiffahrt in beträchtlichem Ausmaß auch in den Alpen betrieben wurde und Flößerei beinahe auf jedem größeren Fluß verbreitet war (Abb. 1). Der Wassertransport war vielfältig und beförderte die unterschiedlichsten Güter auf verschiedenste Weise.

Im Einzugsgebiet der Rhone, die unterhalb des Genfersees selbst nicht mehr in den Alpen liegt, fand wenig Wassertransport statt. Auf ihrem Nebenfluß Isère wurden Eisen aus Allevard und industrielle Produkte der Stadt Grenoble in Richtung Lyon geschifft. Im Gegenverkehr wurden Lebensmittel, vor allem Salz und Getreide, auf Schiffen bergwärts gezogen. Daneben gab es auf einigen Flüssen Flößerei in Richtung unteres Rhonetal und Marseille.

Auf den langgestreckten, nordsüdorientierten Seen der Alpensüdseite konnte sich die Schiffahrt entwickeln, besonders dort, wo die Seen an Alpenpaßrouten lagen. Es gab auch schiffbare Flüsse, besonders hervorzuheben ist die Etsch. Durch die Querverbindung dieser Flüsse und die Verbindung mit dem Po entstanden im Spätmittelalter ein leistungsfähiges Kanalnetz mit der Stadt Mailand im Zentrum und ein weiteres Kanalnetz in Venetien. Beide reichten an einigen Stellen bis an den Alpenrand. Die großen oberitalienischen Städte beeinflußten durch ihren Holzbedarf die Flößerei aus den Alpen in beträchtlichem Ausmaß.

Im Einzugsgebiet des Rheins liegen mehrere Alpenrandseen. Der Wasserweg war hier zum Teil die einzige Verbindung zwischen Alpentälern und Alpenvorland und von entsprechend großer Bedeutung. Besonders stark ausgeprägt war die Schiffahrt auf der Wasserstraße Walensee-Linth-Zürichsee und auf dem Vierwaldstättersee. Der Transitverkehr, die Lebensmittelversorgung, der Transport von Gütern des regionalen Handels und teilweise der Personenverkehr spielten eine wichtige Rolle.

Stefan Brönnimann, dipl. Geograph, Geographisches Institut der Universität Bern, Hallerstraße 12, CH-3012 Bern 


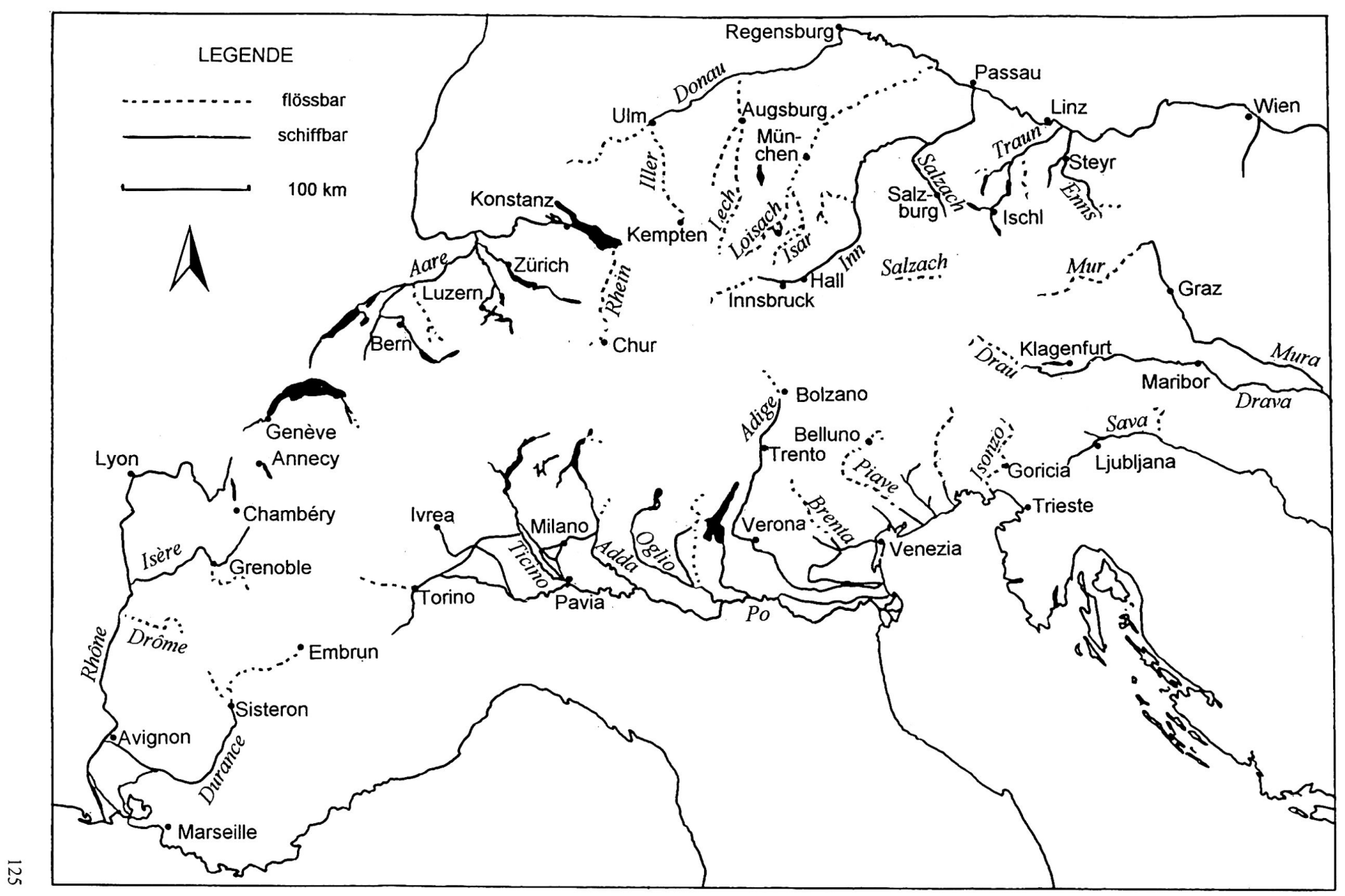

Abb. 1 Karte der schiff- und flößbaren Gewässer in den Alpen von 1500 bis 1800 (nach BRÖNNIMANN 1997). 
Im alpinen Einzugsgebiet der oberen Donau fand die wohl umfangreichste Flußschiffahrt und Flößerei im Alpenraum statt. Grund dafür waren einerseits die zahlreichen Eisenwerke und Salinen, andererseits die großen Städte im Alpenvorland (Augsburg, München, Wien) mit ihren entsprechenden ökonomischen Potentialen. Die wichtigsten schiffbaren Flüsse waren Inn, Salzach, Traun und Enns, die wichtigsten flößbaren Lech und Isar. Eisen, Salz, lokale Erzeugnisse und auch Transitgüter wurden abwärts, Lebensmittel aufwärts geführt.

Im Bereich der unteren Donau war die Schiffahrt etwas weniger intensiv. Sie fand namentlich auf den drei Flüssen Mur, Drau und Sava statt. Auch hier wurden Eisen und Holz abwärts transportiert, und bergwärts wurde Wein und Getreide gezogen. Der Transitverkehr gewann im 18. Jahrhundert an Bedeutung.

Der Massengütertransport spielte gesamthaft wohl die wichtigste Rolle im alpinen Wassertransport. Er war auch geographisch am weitesten verbreitet. Der Export von Holz und Baumaterial aus den Alpen in die Alpenvorlandstädte fand fast auf allen Gewässern statt. Auf einzelnen Gewässern konnten andere Transporte aber eine bedeutendere Rolle spielen und eine größere Dynamik entwickeln: der Export von Salz und Eisen aus den Bergwerken und Salinen der Alpen oder der Transitverkehr.

Der Zusammenhang zwischen der Möglichkeit zur Versorgung auf dem Wasserweg und der Stadtentwicklung ist für die Alpen von MATHIEU (1996) angeschnitten worden. Ein Vergleich der Gewässerkarte (Abb. 1) mit einer Tabelle der Städte in den Alpen vor 1800 zeigt, daß von den wenigen Städten, die es damals in den Alpen gab, mehr als die Hälfte Anschluß an Schiffahrt hatte. Wassertransport war in der Neuzeit in den Alpen im Vergleich zum Flachland aus naturräumlichen Gründen eingeschränkt. Angesichts der Bedeutung des Wassertransports für die Urbanisierung konnte dies für die Alpenstädte einem relativen Entwicklungsnachteil gleichkommen. Innerhalb der Alpen bündelt die Topographie den Verkehr auf wenige Routen. Wassertransport in den Alpen war für die teilhabenden Orte ein Vorteil im Vergleich zu Alpenregionen ohne die Möglichkeit eines Wassertransports.

\section{Das Beispiel Loisach}

Es stellt sich nun die Frage, in welcher Beziehung die Schiff- oder Flößbarkeit eines Gewässers zum Naturraum und zum wirtschaftlichen Umfeld stand. Dies soll vorerst am Beispiel der Loisach in Südbayern erläutert werden.

Die Loisach wurde in der frühen Neuzeit mit Flößen befahren. Die Obergrenze der Floßfahrt war Garmisch (NEWEKLOWSKY 1952), der Endpunkt, nach der Mündung der Loisach in die Isar bei Wolfratshausen, München (Abb. 2). Im 16. und 17. Jahrhundert wurde auf der Loisach Holz und wurden auf den Flößen vermut-

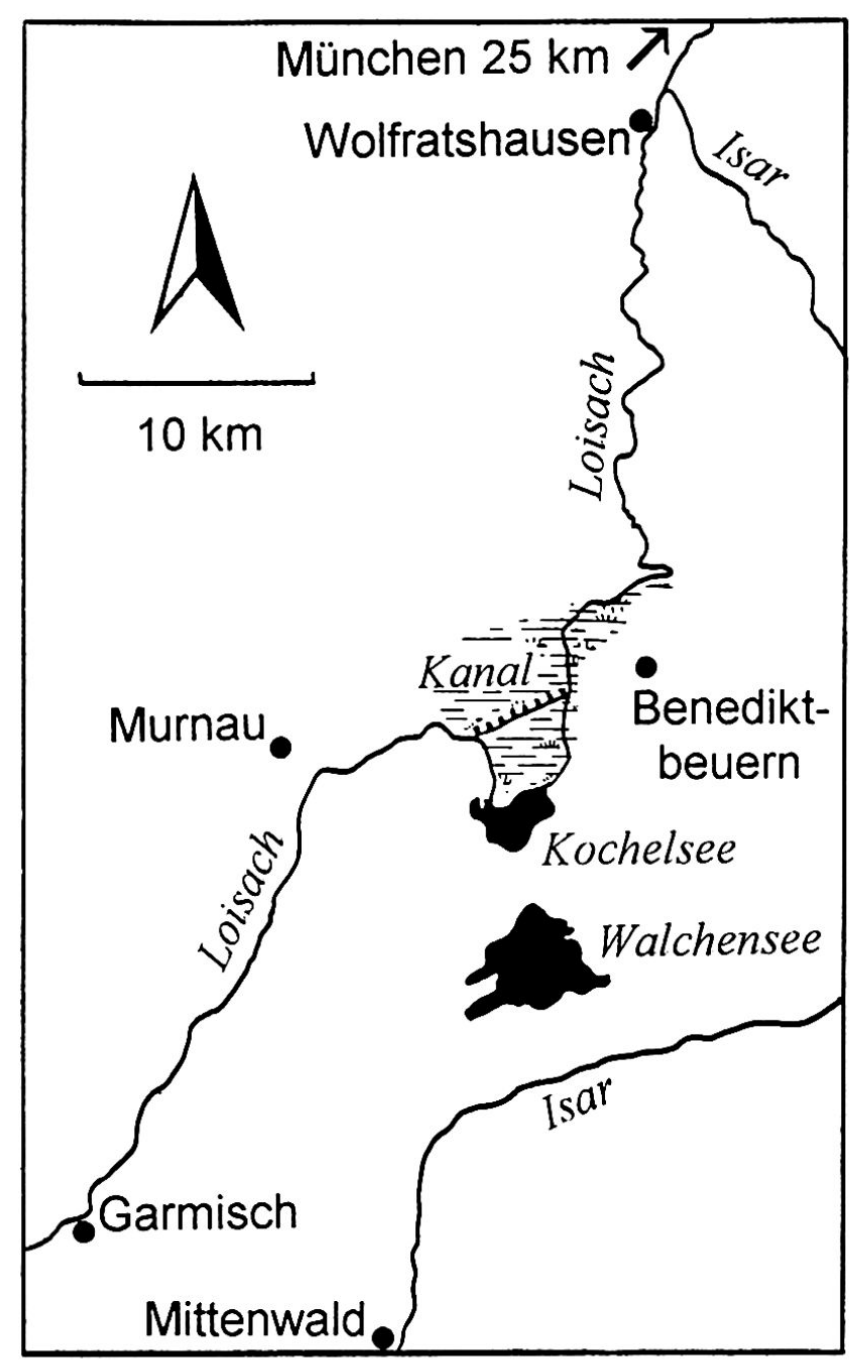

Abb. 2 Der Lauf der Loisach in Südbayern.

lich lokale Produkte und Massengüter zur Versorgung Münchens mit Rohstoffen exportiert. Die Loisach war aber als Wasserweg nicht von überregionaler Bedeutung; hier spielte die Isar eine weit wichtigere Rolle.

Im beginnenden 18. Jahrhundert standen in München zahlreiche Bauvorhaben an. Man benötigte dazu große Mengen der entlang der Loisach gewonnenen Materialien: Marmor, Sandstein, Kalk, Gips, Zement und Schnittholz (vgl. GANZERT und PFADENHAUER 1994, interessant sind auch die zahlreichen sprechenden Ortsund Flurnamen auf den in LINDGREN 1986 reproduzierten zeitgenössischen Karten). Die Güter wurden auf Flößen transportiert. Die Passage durch den Kochelsee bei Benediktbeuern war aber mühsam zu befahren und die richtige Fahrrinne aus dem See schwer zu finden. Unter günstigen Verhältnissen nahm diese Stelle zwei Stunden, sonst bedeutend mehr Zeit in Anspruch. Die Flößbarkeit der Loisach, wie sie bisher bestanden hatte, reichte nun nicht mehr aus. Kurfürst Max Emanuel ließ deshalb 1716 einen Floßkanal durch das Kochelseemoor anlegen. Dieser «Canal zur Floßfarth» war $5 \mathrm{~m}$ breit, 
$50 \mathrm{~cm}$ tief und 4,5 km lang, am Eingang des Kanals befand sich eine Schleuse (LUGAUER 1935). Die Grundbesitzerin, das Kloster Benediktbeuern, widersetzte sich anfänglich der Anlegung des Kanals. Das Kloster befürchtete eine Absenkung des Wasserspiegels, was die Laichplätze der Fische in den flachen Moosseen gefährdet hätte. Diese Einwände konnten aus der Welt geschafft werden, indem das Kloster Gebühren für die Benutzung des Kanals verlangen durfte (NEWEKLOWSKY 1964). Gleichzeitig wurde dem Kloster auch die Unterhaltspflicht übertragen. Mit diesem Kanal war die Flößbarkeit der Loisach unter den neuen, gestiegenen Anforderungen wiederhergestellt. Die Konkurrenz der Straße war zu diesem Zeitpunkt zumindest für den Massengütertransport nicht gegeben (vgl. LINDGREN 1986), um so mehr als der Kanal ganzjährig befahren werden konnte.

Während des 18. Jahrhunderts mußte der Kanal mehrmals geflickt und an einer Stelle verlegt werden. Für das Kloster erwies er sich als finanzielle Belastung. Die Gebühreneinnahmen wurden durch den teuren Unterhalt praktisch aufgezehrt und die tatsächlich stark verminderten Erträge der Fischerei nicht annähernd entschädigt. Als 1752 über eine Verbreiterung des Kanals diskutiert wurde, weigerte sich das Kloster. 1788 kam der Kanal unter staatliche Verwaltung. Er blieb durch das 19. Jahrhundert hindurch in Betrieb, und 1903 wurde sogar noch die Schleuse neu gebaut (LUGAUER 1935).

Das Kochelseemoor zwischen Kochelsee und Kanal wurde extensiv als Streumahd bewirtschaftet (GANZERT und PFADENHAUER 1994). Das Stroh und zuweilen auch Personen wurden im 19. Jahrhundert auf kleinen, nur $5 \mathrm{~m}$ langen Schiffen über den Kochelsee befördert (NEWEKLOWSKY 1952). Der Wasserweg war also unabhängig vom Bauwerk des Kanals für die lokalen Bedürfnisse stets nutzbar gewesen.

\section{Schiff- und Flößbarkeit zwischen Naturraum- ausstattung und wirtschaftlichem Umfeld}

Das Beispiel Loisach zeigt, daß die Möglichkeit des Wassertransports in der Regel nicht «von Natur aus» existierte, sondern zuerst erzeugt und bewirtschaftet, also inwertgesetzt werden mußte. Die Begriffe Schiff- und Flößbarkeit beschreiben Eigenschaften eines Gewässers, die erstens durch dessen wirtschaftliches Umfeld definiert wurden und zweitens technisch weitgehend beeinflußbar waren. Der Kanalbau entsprach der äußersten und sichtbarsten Stufe der Inwertsetzung. Darunter gab es in der frühen Neuzeit eine ganze Anzahl Abstufungen. Seichte Stellen in einem Fluß konnten beispielsweise auf ganz einfache Weise überwunden werden, indem ein flaches Schiff zweimal mit halber Ladung durchgeschleift wurde. Stellen mit nur $20 \mathrm{~cm}$ Wassertiefe konnten so überwunden werden (HOCQUET 1993). Reichte das Durchschleifen der Schiffe nicht mehr aus, konnten die betreffenden Stellen ausgepflügt oder aus- gebaggert oder Felsen im Flußbett gesprengt werden. Stauvorrichtungen konnten durch das Auslösen einer Flutwelle Schiffen die Fahrt auch über längere seichte Strecken ermöglichen. Nur wenig aufwendiger war der Bau von Schleusen. Das Inventar zeigt, daß auch in den Alpen erstaunliche Hindernisse überwunden werden konnten. Die naturgeographischen Verhältnisse wurden somit bereits in der frühen Neuzeit zu einer Kostenfrage. Auch wenn keine Wasserbauten nötig waren, konnten Schiff- und Flößbarkeit stets nur durch großen Arbeitsaufwand aufrechterhalten werden. Die Bauten selbst verlangten, wie das Beispiel zeigt, kostspieligen Unterhalt. Die Schiff- oder Flößbarkeit eines Gewässers mußte dauernd reproduziert werden (vgl. auch die auf der Titelseite abgebildete einfache Vorrichtung zur Erleichterung der Bergfahrt). Nutzungskonflikte, gezeigt durch den anfänglichen Widerstand des Klosters, konnten zusätzlich eine wichtige Rolle spielen.

In einem verkehrshistorischen Kontext interessiert die Inwertsetzung von Schiff- und Flößbarkeit in einem sich dauernd verändernden wirtschaftlichen und technischen Umfeld. Gerade in den Alpen befand sich Schiffahrt oder Flößerei in einem Grenzbereich. Die Frage nach den Grenzen der Inwertsetzung von Schiff- oder Flößbarkeit kann bei allen Alpengewässern und für jede Zeitspanne gestellt werden.

\section{Die Entwicklung des Wassertransports in den Alpen}

Die Entwicklung des Wassertransports in den Alpen während der Neuzeit kann anhand dieser Frage folgendermaßen beschrieben werden. Auf den meisten Flüssen schob sich die Grenze der Schiffbarkeit im Verlauf der Zeit dem Flachland zu, am weitesten verbreitet war die Nutzung vermutlich im Spätmittelalter und zu Beginn der Neuzeit. Im damals herrschenden wirtschaftlichen und technischen Umfeld war der Wasserweg nicht nur im Flachland und nicht nur für Massengüter dem Landweg überlegen. Entsprechend war auch die Vielfalt der transportierten Güter am größten. Auf einigen Flüssen spielte dabei auch die Konjunktur der Bergwerke eine Rolle. Bereits im 17. Jahrhundert zeichnete sich mancherorts Stagnation ab, während sich der Wassertransport auf anderen Flüssen noch weiter entfalten konnte. Mit dem Aufkommen der Straßen beschleunigte sich im Verlauf des 18. Jahrhunderts der Strukturwandel im Wassertransport. Die Verschiebung der Obergrenze der Schiffahrt ging mit einer Verschiebung des Transportguts in Richtung Massengüter einher. Während die Schiffahrt auf einigen Flüssen einging, wurde die Flößerei immer umfangreicher. Im Flachland wurde in dieser Zeit der Wassertransport durchaus als zukunftsträchtige Transportart betrachtet, wie der Ausbau des französischen Binnenwasserstraßennetzes im 17. bis 19. Jahrhundert zeigt. Im Alpenraum überwogen aber meist andere Interessen, und auch der Holztransport vollzog sich zunehmend nur noch durch Trift 
(das Schwemmen von losen Baumstämmen, oft auch ungenau als Flößerei bezeichnet). Auf den Seen setzte eine andere Entwicklung ein. Die Schiffahrt auf den Seen konnte vom Ausbau der Straßen im Alpenvorland unter Umständen sogar profitieren und schaffte später mit dem Dampfschiff und dem beginnenden Tourismus den Anschluß an das Industriezeitalter. Damit fand eine erneute Inwertsetzung statt.

Die Stagnation und der Niedergang des Wassertransports in den Alpen im 18. Jahrhundert kann auch als Teil eines europäischen Konzentrations- und Spezialisierungsprozesses im Transportwesen verstanden werden, bei welchem der Wassertransport vorerst noch seinen Platz erhielt. Die Alpen jedoch verloren in diesem Prozeß den Anschluß an das Wasserstraßennetz mehr und mehr.

\section{Literatur}

BERGIER, J.-F. (1974/75): Le trafic à travers les Alpes et les liaisons transalpins du haut Moyen Age au XVIle siècle. In:
Le Alpi e Europa, Bd. 3 (Atti del Convegno di studi, Milano, 1973). Bari.

BRÖNNIMANN, S. (1997): Die schiff- und flößbaren Gewässer in den Alpen von 1500 bis 1800 . Versuch eines Inventars. In: Der Geschichtsfreund Bd. 150, S. 119-178.

GANZERT, Ch., PFADENHAUER, J. (1994): Geschichte der landwirtschaftlichen Entwässerung am Beispiel der Loisach-/Kochelseemoore. In: Deutscher Verband für Wasserwirtschaft und Kulturbau e. V. (Hrsg.) Historische Wasserwirtschaft im Alpenraum und an der Donau. Stuttgart. S. 271-289.

HOCQUET, J.-C. (1993): Weißes Gold. Das Salz und die Macht in Europa von 800 bis 1800. Stuttgart.

LINDGREN, U. (1986): Alpenübergänge von Bayern nach Italien 1500-1850. München.

LUGAUER, F. K. (1935): Der Floßkanal im Benediktbeurer Moos. In: Lech-Isar-Land, Monatsschrift des Heimatverbandes Huosigau, Dießen. Nr. 11 (1), S. 4-8.

MATHIEU, J. (1996): Urbanisierung in den Alpen von 1500 bis 1800. Fakten und Thesen. In: KÖRNER, M. und WALTER, F. (Hrsg.) Quand la montagne aussi a une histoire. Mélanges offerts à Jean-François Bergier. Bern. S. 337-360.

NEWEKLOWSKY, E. (1952, 1964): Die Schiffahrt und Flößerei im Raume der oberen Donau. 3 Bände. Linz. Band 11952 , Band 31964. 